\title{
EVACUATION PLANNING USING THE INTEGRATED SYSTEM OF ACTIVITY-BASED MODELING AND DYNAMIC TRAFFIC ASSIGNMENT
}

\author{
Dung-Ying Lin ${ }^{*}$ \\ The University of Texas at Austin, \\ Department of Civil, Architectural \& Environmental Engineering \\ 1 University Station, C1761, Austin, TX 78712 \\ Phone: (512) 471-4539; Fax: (512) 475-8744; E-mail: dylin@mail.utexas.edu \\ ${ }^{*}$ Corresponding Author
}

\section{Naveen Eluru}

The University of Texas at Austin, Department of Civil, Architectural \& Environmental Engineering 1 University Station, C1761, Austin, TX 78712

Phone: (512) 471-4535; Fax: (512) 475-8744; Email: naveeneluru@mail.utexas.edu

\section{S. Travis Waller}

The University of Texas at Austin, Department of Civil, Architectural \& Environmental Engineering 1 University Station, C1761, Austin, TX 78712

Phone: (512) 471-4539; Fax: (512) 475-8744; E-mail: stw@mail.utexas.edu

\section{Chandra R. Bhat}

The University of Texas at Austin, Department of Civil, Architectural \& Environmental Engineering 1 University Station, C1761, Austin, TX 78712 Phone: (512) 471-4535; Fax: (512) 475-8744; Email: bhat@mail.utexas.edu 


\begin{abstract}
The occurrence of natural disasters in the coastal regions and numerous potential events within urban regions has drawn considerable attention among transportation stakeholders. Federal, state and local officials need to be effectively prepared to address the challenges raised by an evacuation. The focus of this research effort is to develop a tool to study the repercussions of evacuation of an entire regional transportation network recognizing the human behavior element. Neglecting these seemingly chaotic traffic flow patterns would lead to inaccurate system assessment and predictions. We study the influences of evacuees' locations in the urban region at the moment of emergency alert. In addition, we identify the locations of all the members of the household and explicitly consider household member interactions. Further, we study the accurate times the individuals enter the network to evacuate the study region, which can vary based on where the other household members are located at that time and the travel time on the network to reach these locations. To accomplish the goals, we employ the integration framework of activitybased modeling and dynamic traffic assignment to study the evacuation traffic flow patterns at the time of evacuation. Specifically, the paper formulates the evacuation problem and discusses the utility of deploying the integrated module of activity-based modeling and dynamic traffic assignment for evacuation planning and outlines the challenges in integrating these two tools.
\end{abstract}




\section{INTRODUCTION}

The occurrence of natural disasters and other extreme events in coastal and urban regions (such as Hurricane Katrina and Hurricane Rita) has drawn attention to developing emergency evacuation plans aimed at reducing loss of life and property and coping with the immediate societal consequences [see (1) and (2)]. An important part of these evacuation plans is the mobilization of federal, state and local agencies in a timely fashion, but also important is the provision of resources and knowledge to these agencies to implement effective evacuation strategies. In the latter context, it is important to understand and model human response behavior and transportation flow operations, and especially the interplay between the two, once an emergency evacuation alert is issued.

The objective of the current research effort is to develop a tool to study and model evacuation impacts on the transportation network, while recognizing aspects of human response behavior that are likely to be manifested during such evacuation calls. To effectively achieve this objective, it is critical to accurately model traffic flows on the network, which will be influenced by the locations of individuals in the urban region at the moment of the emergency alert. However, it is not enough simply to spatially and temporally locate all the individuals in the urban area; rather, it is important to map and associate the spatial positions of individuals based on such relationships as whether individuals belong to the same household or not. This is because household members would attempt to gather together and evacuate the region as a single unit [see (3-7)]. In doing so, it is possible that some household members would travel in directions opposite to the direction of evacuation. Neglecting these seemingly haphazard traffic flows would lead to inaccurate traffic flow predictions. Further, it is also important to accurately identify the times when, and places where, individuals enter different parts of the transportation network in response to an evacuation alert. These spatial-temporal paths will depend not only on the evacuation strategies of individual households (in terms of who picks up whom, and/or or the location to assemble), but also on the travel times in the network. But the travel times themselves depend on how the response of individual households collectively influences network performance, while the precise individual household evacuation strategies may evolve depending on perceived network performance. This is, of course, a classic case of demand-supply interaction, or the traffic assignment problem. However, a particular need in an emergency evacuation context is that the dynamics of demand and traffic flows be considered along a fine resolution of time (that is, in seconds and minutes, rather than on an hourly or peak/off-peak period basis).

To summarize, the preceding discussion highlights two important aspects of modeling traffic flows in the aftermath of an emergency alert to evacuate from an urban region. First, there is a need to accurately predict the locations of individuals at the instant the emergency alert is provided (with information on the location of other household members also available). Second, there is a need to predict the travel times on network links at a fine resolution along the time dimension (affected by the evacuation exit points, the locations of household members, and the evacuation strategies of individual households).

In the current paper, we address the two points above through the integration of an activity-based model and a dynamic traffic assignment model. Lin et al. (2008) propose such an integrated framework for general regional planning (8), and we customize their framework in this paper to 
modeling traffic flows in an emergency evacuation context. The paper formulates the evacuation problem, discusses the value of deploying an integrated ABM-DTA system for evacuation planning, and implements the system using a network sampled from the Dallas-Fort Worth region.

The rest of the paper is organized as follows. Section 2 discusses the ABM and DTA approaches and their applicability to evacuation planning. Section 3 outlines the conceptual framework, with an emphasis on the assumptions made in the process of integrating the ABM and DTA modules. Section 4 presents the results of the application of the integrated tool for a test network. Section 5 concludes the paper highlighting the lessons learned and limitations of the research.

\section{ABM and DTA FRAMEWORK - APPLICABILITY TO EVACUATION PLANNING}

For nearly thirty years, the traditional trip-based approach to transportation modeling has dominated the planning process. However, the trip-based approach is saddled with many limitations (for example, see (9-14). This has led to an active stream of research that examines alternative paradigms for predicting travel demand and supply by incorporating more behaviorally realistic methodologies. These research attempts have resulted in the development of ABM and DTA frameworks.

\subsection{ABM}

On the demand side of transportation modeling, researchers have attempted to overcome the conceptual and behavioral inadequacy of the trip-based approach through the use of an activitybased modeling (ABM) paradigm. Activity-based approaches to modeling travel demand are conceptually more appealing compared to the trip-based method for the following reasons: (1) Treatment of time as a continuum and a generally superior incorporation of the temporal dimension, (2) Focus on sequences and patterns of activities and travel (i.e., tours) rather than individual trips, (3) Recognition of linkages among various activity-travel decisions, (4) Incorporation of intra-household interactions, inter-personal and intra-personal consistency measures, (5) Consideration of space-time constraints on activities and travel, and (6) Emphasis on individual level travel patterns. The potential benefits of the activity-based analysis and the resulting interest in operationalizing the activity-based approach have sparked an interest in micro-simulation based modeling systems. A number of micro-simulation platforms that employ the activity-based paradigm of transportation demand forecasting have been developed recently, such as CEMDAP [see (13) and (15)], FAMOS [see (38)], and the model systems designed for Portland METRO [see (16)], New York NYMTC [see (17)], Columbus MORPC [see (18)], Sacramento SACOG [see (19)] and the San Francisco SFCTA [see (20)]. Activity-based models, with their inherent advantages over the trip-based models, lend themselves naturally to addressing the evacuation problem.

\subsection{DTA}

On the supply side of transportation modeling, conventional techniques of trip assignment based on static traffic assignment (STA) have been employed for decades. The limitations of the static assignment procedures and the increase in computing capacity have allowed the field to move toward more behaviorally realistic dynamic traffic assignment (DTA) models. DTA techniques 
offer a number of advantages relative to the STA methods including: (1) Capturing timedependent interactions of the travel demand and supply of the network, (2) Capability to capture traffic congestion build-up and dissipation, (3) Accommodating the affect of ramp-meters and traffic lights on the network are more straightforward, (4) Suited to model the effects of ITS technologies and (5) The network representation can be undertaken at a disaggregate level. A number of simulation-based DTA modules have been developed in the recent past such as VISTA [see (21)], CONTRAM [see (22)], DynaMIT [see (23-25)] and DYNASMART-P [see (26)].

\subsection{Applicability to evacuation planning}

In this section, we focus on the CEMDAP ABM model system and the VISTA DTA model system, and discuss the integration of these model systems for application to traffic modeling after evacuation alerts.

\subsubsection{CEMDAP}

The Comprehensive Econometric Micro-simulator for Daily Activity-travel Patterns (CEMDAP) is a micro-simulation implementation of a continuous-time activity-travel modeling system, proposed by Bhat et al. (13). CEMDAP takes as input information on the aggregate socioeconomics and the activity-travel environment characteristics in the urban study region for the base year, as well as policy actions being considered for future years (the activity-travel environment includes the land-use, urban form, and transportation level-of-service (LOS characteristics). The aggregate-level base year socioeconomic data are first fed into the synthetic population generator (SPG) to produce a disaggregate-level synthetic dataset describing a subset of the socioeconomic characteristics of the households and individuals residing in the study area (see (27) for information on the SPG module). Additional base-year socioeconomic attributes related to mobility, schooling, and employment at the individual level, and residential/vehicle ownership choices at the household level, that are difficult to synthesize (or cannot be synthesized) directly from the aggregate socioeconomic data for the base year are simulated by the Comprehensive Econometric Microsimulator for SocioEconomics, Land-use, and Transportation System (CEMSELTS), (see (28) for more details). The base year socioeconomic data, along with the activity-travel environment attributes, are then run through CEMDAP to obtain individual-level activity-travel patterns (see (13) and (15) for details). The activity-travel patterns are subsequently passed through a dynamic traffic micro-assignment scheme to determine path flows, link flows, and transportation system LOS by time of day. In the framework, the initial iteration of CEMDAP needs the LOS values as inputs. However, the values used in the iteration need not be the "true" LOS values. So it is necessary to rerun the CEMDAP module with the new LOS variables obtained.

\subsubsection{VISTA}

The Visual Interactive System for Transport Algorithms (VISTA) is a comprehensive DTA system that integrates data warehousing and traffic analysis for transport applications via a client-server implementation. VISTA was originally outlined in Waller and Ziliaskopoulos (21). As with many contemporary simulation-based DTA approaches, VISTA is comprised of three primary modules: traffic simulation, time-dependent routing algorithms, and path assignment. 
The traffic simulator in VISTA is RouteSim [see (29)], a route-based traffic simulator based on the Cell Transmission Model [see (30-31)]. RouteSim takes a network (nodes, links and controls) as well as the spatial path assignment as input and outputs the spatio-temporal trajectories of travelers. The time-dependent shortest path (TDSP) module is implemented according to Ziliaskopoulos and Mahmassani [see $(32,33)$ ] and has substantial potential for distributed and parallel implementations (Ziliaskopoulos and Kotzinos, (34)) which is critical for large-scale deployments.

Path assignment in VISTA is handled through multiple means. The traditional MSA approach is employed for early iterations, but gap function based methods are employed to obtain meaningful convergence in later iterations. For the latter a variety of gap functions are employed which are based on the variational inequality formulation as detailed in Chang (35).

VISTA typically employs time-scales of approximately 6 seconds for traffic dynamics (for simulation, time-dependent routing, and trip departure times). A scale of approximately 5 minutes is common for path choice behavior (i.e., travelers departing within 5 minutes of each other between the same origin-destination pair will observe similar conditions). It should be noted that this minor 5-minute aggregation occurs after TDSPs have been found based on the 6 second scale.

The path assignment and TDSP modules were reengineered into an efficient module that can handle large data sets in Ziliaskopoulos and Waller (36). Ziliaskopoulos et al. (37) developed an Internet-based geographic information system (GIS) and incorporated it into the system framework. This equipped VISTA with the unique feature of being accessed over the Internet via web browser, CORBA interface or Java GIS. The feature eliminates the need for software installation/upgrade and allows users to conveniently access the consistent analysis without spatial limitation.

\subsubsection{Applicability}

An integrated tool employing ABM (CEMDAP) and DTA (VISTA) modules offers the required spatial, temporal and human behavior information essential for evacuation planning. To elaborate, the CEMDAP module provides the spatial and temporal locations of individuals (and other household members) at any give time of the day. In addition to the spatial temporal details, information on individual modal accessibility is also available. These individual level details are used to develop a string of origin-destination trips (with detailed mode and time of day information) each individual would make in order to exit the study region. The VISTA module, based on this information, loads the origin-destination trips on the transportation network and allows us to compute accurate measures of travel time and traffic congestion resulting from travel for evacuation.

An important point regarding the applicability of the CEMDAP-VISTA system is the temporal fidelity built into the integrated system. In particular, the timing of issue of an emergency evacuation alert is critical in evacuation planning. The activity travel patterns of individuals (and implicitly their locations) are dependent on the time of day. For example, at 3 AM in the night, a large part of the population would be in their residences. However, at 12 Noon in the day, the individuals of each household are more likely to be dispersed at distinct locations in the urban 
area. So, any model of evacuation needs to consider the time of day at a high level of temporal resolution. Also, the dynamics of traffic flow following the alert needs to be considered at a fine level of temporal resolution. Thus, the integrated system of ABM and DTA framework, with a strong emphasis on a fine resolution of time of day, provides a suitable tool to analyze the evacuation planning process.

\section{FRAMEWORK FOR EVACUATION PLANNING}

In this section we discuss the details of the framework employed in the analysis. Section 3.1 details the rules proposed to model individuals' behavior in the event of an emergency alert. These rules allow us to generate the time dependent OD matrix for loading on the transportation network. Section 3.2 presents the implementation details of loading the OD matrices on the transportation network.

\subsection{Proposed rule set}

The ABM module provides the exact temporal and spatial coordinates of individuals in the urban region. These coordinates can be translated into time-dependent OD matrices that serve as input into the DTA module. However, as highlighted earlier, it is not adequate to simply locate individuals. The temporal and spatial information of all the members of the household needs to be mapped and associated to accurately generate the evacuation pattern for individuals and households (as a single unit). The evacuation pattern is itself a function of the underlying evacuation strategy adopted by households in response to an emergency alert to leave the urban region. While the evacuation strategy of households is an area for research study, we use a set of intuitive rules in the current paper to represent this strategy. The set of rules attempts to simulate individual response behavior based on the spatial, temporal, and mobility constraints of individuals and households. Explicit consideration is given to whether or not households have children in the formulation of the rules, since children lack mobility and depend on other household members to be evacuated. The rules are discussed in the next two sections.

\subsubsection{Households without children}

In households without children, the predominant constraint on travel for evacuation is driven by auto access. The following guidelines are proposed for determining travel plans for different members of the household: (1) If all adults have auto access, they leave the urban region independently, (2) If there are multiple, but not all, adults with auto access, the nearest adult with auto access will pick up the adult without auto access, (3) If the household has only one auto available, the adult with auto access will pick all the others and (4) If the household does not have auto access, we assume a special auto (taxi or dial-a-ride) will pick up individuals.

\subsubsection{Households with children}

In households with children, the individuals' behavior is centered on children and auto availability. The following guidelines are proposed for determining travel plans for different members of the household: (1) If all adults in the household have auto access, the task of picking up children is distributed among the adults, (2) If the number of autos in the household is less than the number of adults, the task of picking up is distributed among those with auto access, with emphasis on picking up the nearest person first, (3) If there is only one adult in the 
household, he/she will pick up all the children and evacuate and (4) If the household does not have auto access, we assume a special auto (taxi or dial-a-ride) will pick up individuals.

\subsubsection{Summary}

The rules proposed above are developed to facilitate the modeling of individual response decisions and travel patterns. The rules, by no means, comprehensively tackle all the potential situations that individuals might encounter. Instead, the rule-based system simply provides a flexible and practical framework that can track the movement of each individual in the network during evacuation. The rules, of course, can be modified when the analyst has more information on actual behavioral responses of individuals to emergency evacuation alerts.

\subsection{Assignment implementation}

In this section, we discuss the assignment implementation details of the evacuation problem. Specifically, using the list of each individual's stops (generated based on the rule framework proposed), we determine the order and departure time at each stop by loading the O-D trips on the network. The actual travel time to the destination is affected by the network characteristics and actual travel time, thus impacting the travel start time for the next stop. These impacts trickle down across the network and affect the network travel times significantly. So, it is necessary to consider these details in the implementation. To elaborate, consider the case of a single person who needs to make two stops to pick-up household members prior to evacuating the region. The order of these stops is determined based on an individual's perceived travel time. Let's suppose the person begins his or her travel from $\mathrm{O}$ and wants to reach $\mathrm{D}$. The trip chain will look like O1-2-D where 1, 2 represent his or her planned stops. In order to start the network loading we assume a perceived travel time for each segment of the trip chain and accordingly load them on the network. So, based on perceived times, let the dynamic trip chain and departure times be $\mathrm{O}$ $(9: 00 A M)-1(9: 10 A M)-2(9: 30 A M)$ - Destination (10:00 AM), which is translated as three dynamic OD trips with departure times of 9:00AM, 9:10AM and 9:30 AM respectively. Once the network is loaded with these trips (and trips corresponding to all travelers), the actual segment travel times are obtained based on the network flows. In the next iteration, suppose we find that the travel time for segment 1-2 is 30 minutes, instead of the assumed 20 minutes due to the evacuation traffic. Then the dynamic trip for this person has to be modified to O (9:00 AM) -1 $(9: 10 A M)-2(9: 40 A M)$ - D (10:10 AM) in the second iteration. Similarly, the dynamic OD tables of all travelers should be modified accordingly and loaded into a DTA module to reevaluate. In other words, there is a need for an iterative procedure to arrive at a correct dynamic OD table that should result in the correct travel time.

Let $\xi(\tau)$ be the dynamic OD table with the vector of link travel time $\tau$ and let $\Psi$ be the dynamic traffic assignment module. The fixed-point formulation of the dynamic OD table and link travel time can be formulated as:

$$
\tau=\Psi(\xi(\tau))
$$


To address this fixed-point problem in Equation (1), the following iterative procedure is proposed $^{1}$ :

Step 1: Retrieve destinations based on activity pattern predictions

Step 2: Find the optimal trip for each person based on the perceived link travel times.

Step 3: Generate dynamic OD table based on the current trips.

Step 4: Load the OD table obtained in Step 3 on the transportation network and run DTA.

Step 5: Retrieve link travel time from DTA results.

Step 6: Update travel time $\tau_{l}$ of link $l$ with Method of Successive Average (MSA)

$$
\tau_{l}^{\text {new }}=\frac{1}{\text { iteration }} \times \tau_{l}^{\text {iteration }}+\frac{\text { iteration }-1}{\text { iteration }} \times \tau_{l}^{\text {iteration }-1}
$$

Step 7: If the travel time converge, stop. Otherwise, go back to step 3.

In step 2, we generate the optimal trip for each individual. So, in the example discussed earlier, , we enumerate the trip combinations (O-1-2-D and O-2-1-D in this case) and find the trip with minimal cost. However, the enumeration may not necessarily alter the results substantially because it is difficult for users to make perfectly rational decision during an evacuation process. In the study, we enumerate the possibilities for demonstration purpose. In large-scale evacuation planning process, these steps can be replaced by random assignment to reduce the computational effort.

It is important to note that the process detailed above essentially estimates the dynamic OD table during evacuation. We can plot these OD tables as vehicle loading and departure curves that allow us to exactly identify locations and times of vehicles in the network. The dynamic OD table is of critical importance for government agencies when predicting traffic flows within the network, or for conducting traffic analysis or evaluating evacuation management measures.

\section{NUMERICAL EXPERIMENTS}

The integrated tool developed in the research effort was tested with two primary objectives. The first is to understand the impact of time of day on evacuation traffic patterns. The focus is on studying whether the timing of the evacuation alert influences the system evacuation time significantly. The second is to analyze the impact of the population size of the evacuation zone on the evacuation system performance.

\footnotetext{
${ }^{1}$ The reader should note here that for the current study, we assume the free flow travel times as the perceived travel times between each OD pair.
} 
The measures employed for evaluating the system performance include (1) Total System Travel Time (TSTT) and (2) Network Clearance Time (NCT) . TSTT is the sum of travel times of all the vehicles that are loaded on the network in the proces of evacuation. The NCT time is defined as the difference between the time when the last vehicle leaves the evacuation network to the time when the evacuation alert has been issued. The TSTT is typically employed as a performance index when considering network improvements. If the goal of the planning process is to evacuate people from the study region in the smallest possible time, NCT is a preferred performance measure. However, both measures in conjunction can yield insights on the network performance. So, for the purpose of the experiments, we employ both measures. In addition, we employ the loading curve and departure curve for ease of understanding. The loading curve reprsents the cumulative number of vehicles that enters the network at each point in time after the emergency evacuation alert is issued, while the departure curve represents the cumulative number of vehicles that leave the network as a funcion of time from the issue of the alert. In the study, we assume that, when an evacuation alert is issued, all evacuees in the network respond instantaneously. It is straightforward to incorporate a varying response time profile in the proposed framework if needed.

The framework is tested on a sampled network. The demographic information (including household size, number of adults, number of children, household vehicles, employment and school location) required for CEMDAP is obtained by sampling the Dallas Fort Worth demographic data generated using SPG and CEMSELTS (see (28) for more details) . The network data essential for VISTA are obtained by sampling data sets provided by North Central Texas Council of Government. In addition to the physical network, we include an additional super sink node to represent exit from the study region. The super sink node is connected to five chosen candidate exit nodes (57,717, 57,784, 58,805, 58,033 and 57,864). With the single destination network, we can also determine the evacuation exits and analyze the flow patterns. The running environment of VISTA is Linux with an Intel 3.00GHz CPU and 32 GB memory; while the environment of CEMDAP is Windows XP with Intel 3.4 GHz CPU and 2 GB memory. The experimental network is depicted in FIGURE 1. 


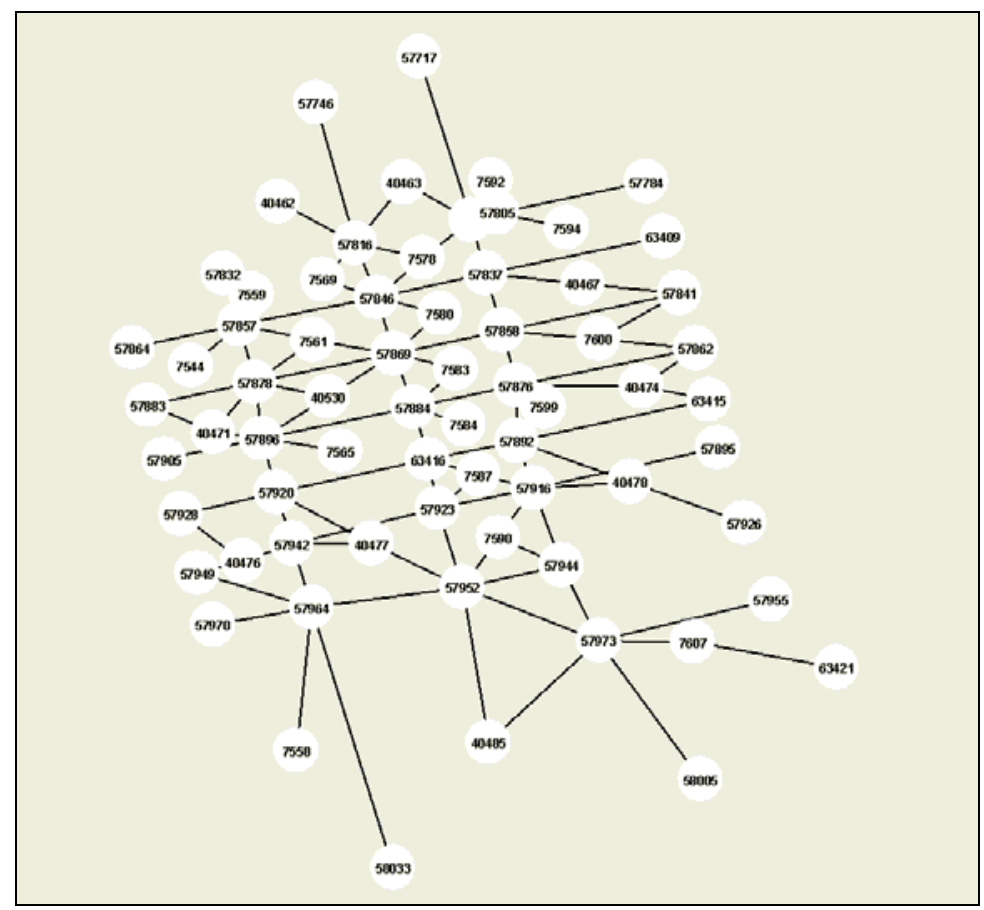

FIGURE 1: Grid Network

In the first experiment, we consider the scenario of 1,000 households and an evacuation alert issued at 9AM. Totally, the number of trips generated after considering household interactions is 1,529. By applying the framework proposed in this study, the TSTT and NCT are 2,011,765 and 5,568 seconds, respectively. FIGURE 2 provides the loading and departure curves for this scenario. Note that the area between the two curves represents the TSTT with the current demand and supply level.

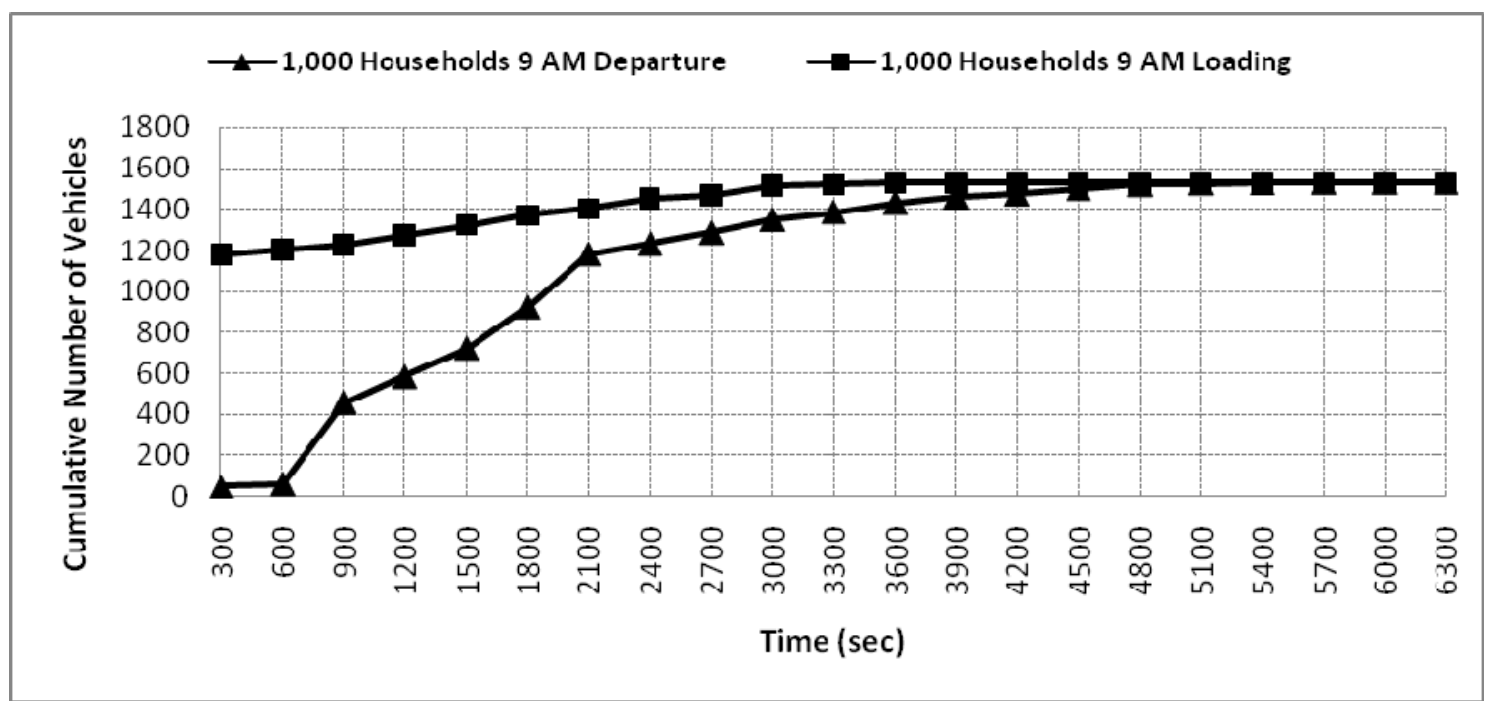

FIGURE 2: 1,000 Households Network Loading and Departure Curve

The information in Figure 2 provides important information that can aid in evacuation planning. For instance, by drawing a vertical line at 1,200 seconds since the issuance of the evacuation alert, we know that 691 vehicles are present in the network (1,276 vehicles loaded, while only 
585 vehicles have left the network). In addition, by drawing a horizontal line at the value of 1,200 for cumulative number of vehicles, we note that it takes roughly 2,100 seconds to evacuate 1,200 vehicles.

Next, we consider 5,000 households at the same time of day. The number of trips is 7,414. The TSTT and NCT measures are 10,216,626 and 5,886 seconds respectively. FIGURE 3 provides the loading and departure curves for this scenario. The same analysis applies in this scenario as in the 1,000-household scenario.

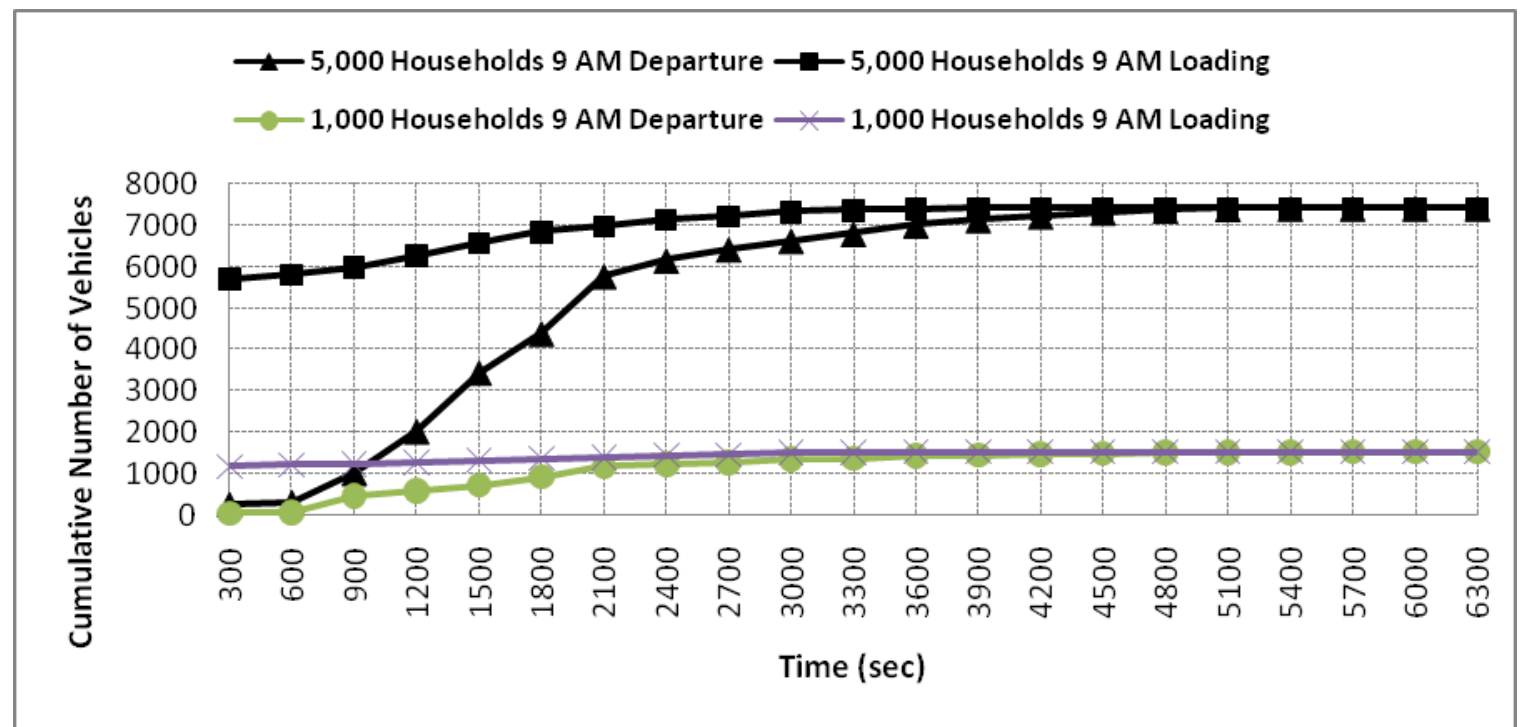

FIGURE 3: 5,000 Households Network Loading and Departure Curve

In FIGURE 3, we also include the loading and departure curves for the 1,000 households scenario for comparison purposes. As can be observed from the figure, the main difference between the two scenarios is the departure curve. Compared to the 5,000 households scenario, the 1,000 households scenario has a relatively flat departure curves since the 1,000 households do not generate heavy evacuation traffic. On the other hand, an apparent surge can be observed in the 5,000 scenario between 900 seconds and 2,100 seconds. If traffic management measures are to be adopted in the 5,000 households scenario during evacuation planning period, this time period could be potentially a good choice.

\subsection{Impact of Household Number}

To further understand the impact of household numbers on the evacuation system, we conduct one more test with 25,000 households at the same time of day. The comparison is presented in TABLE 1.

TABLE 1: Impact of Household Number at 9 AM

\begin{tabular}{|c|c|c|c|}
\hline $\begin{array}{c}\text { Number of } \\
\text { Households }\end{array}$ & $\begin{array}{c}\text { Number of Evacuation } \\
\text { Trips }\end{array}$ & $\begin{array}{c}\text { Total System Travel } \\
\text { Time (sec) }\end{array}$ & $\begin{array}{c}\text { Network Clearance } \\
\text { Time (sec) }\end{array}$ \\
\hline \hline 1,000 & 1,529 & $2,011,765$ & 5,568 \\
\hline 5,000 & 7,414 & $10,216,626$ & 5,886 \\
\hline 25,000 & 36,062 & $93,968,010$ & 7,464 \\
\hline
\end{tabular}


Note that when the number of households increases from 1,000 to 5,000, the NCT and TSTT increase by $5.71 \%$ and $407.84 \%$, respectively. However, the network clearance time and network clearance time increase by $26.81 \%$ and $819.76 \%$ respectively when the number of households increased from 5,000 to 25,000 . The non-linear congestion effect can be observed from the results.

\subsection{Impact of Time of Day}

In this example, we demonstrate the impact of the time of day when the emergency alert is issued, using the 1,000-household scenario. The results are summarized in TABLE 2:

TABLE 2: Impact of Time of Day for 1,000 Households Scenario

\begin{tabular}{|c|c|c|c|}
\hline Time & $\begin{array}{c}\text { Number of Evacuation } \\
\text { Trips }\end{array}$ & $\begin{array}{c}\text { Total System Travel } \\
\text { Time (sec) }\end{array}$ & $\begin{array}{c}\text { Network Clearance } \\
\text { Time (sec) }\end{array}$ \\
\hline 9 AM & 1,529 & $2,011,765$ & 5,568 \\
\hline 12 PM & 1,795 & $2,413,856$ & 6,672 \\
\hline 4 PM & 1,911 & $2,691,764$ & 6,330 \\
\hline
\end{tabular}

As can be observed from the table, the time required to evacuate the network at $12 \mathrm{PM}$ is $19.83 \%$ more than the time at 9 AM. The TSTT values differ by 111.69 hours (402,091 seconds) between the two times of day. At 4 PM, more activities take place in the network. Hence, the TSTT reaches its highest level among the three cases tested. However, the reduction in NCT at 4 PM compared to 12 Noon is because individuals of the same household are more likely to be dispersed in space at noon than at $4 \mathrm{pm}$, requiring more intra-urban trips for assembling together as one unit before heading out of the urban region. The loading curves and departure curves for various times of day are depicted in FIGURE 4 and FIGURE 5 respectively.

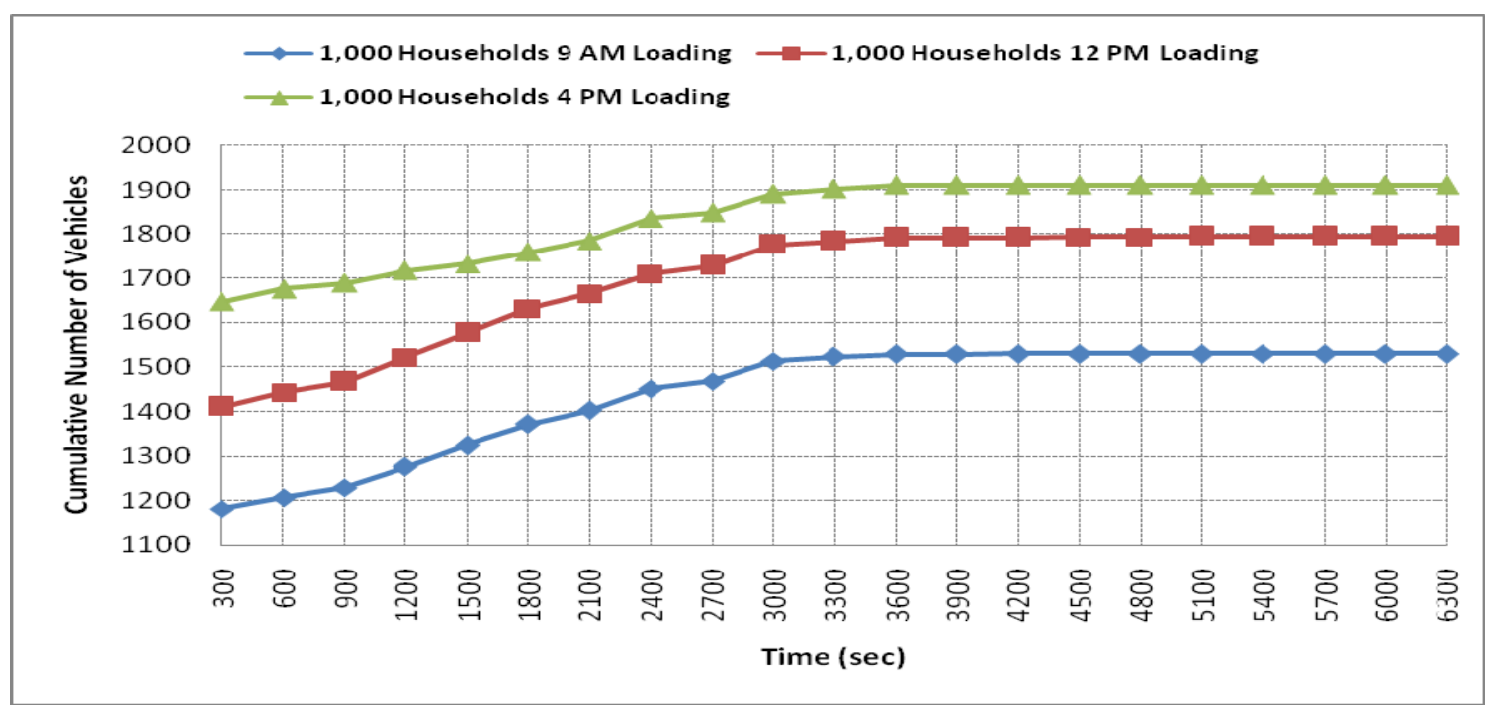

FIGURE 4: The Loading Curves for 1,000 Households at Various Times 


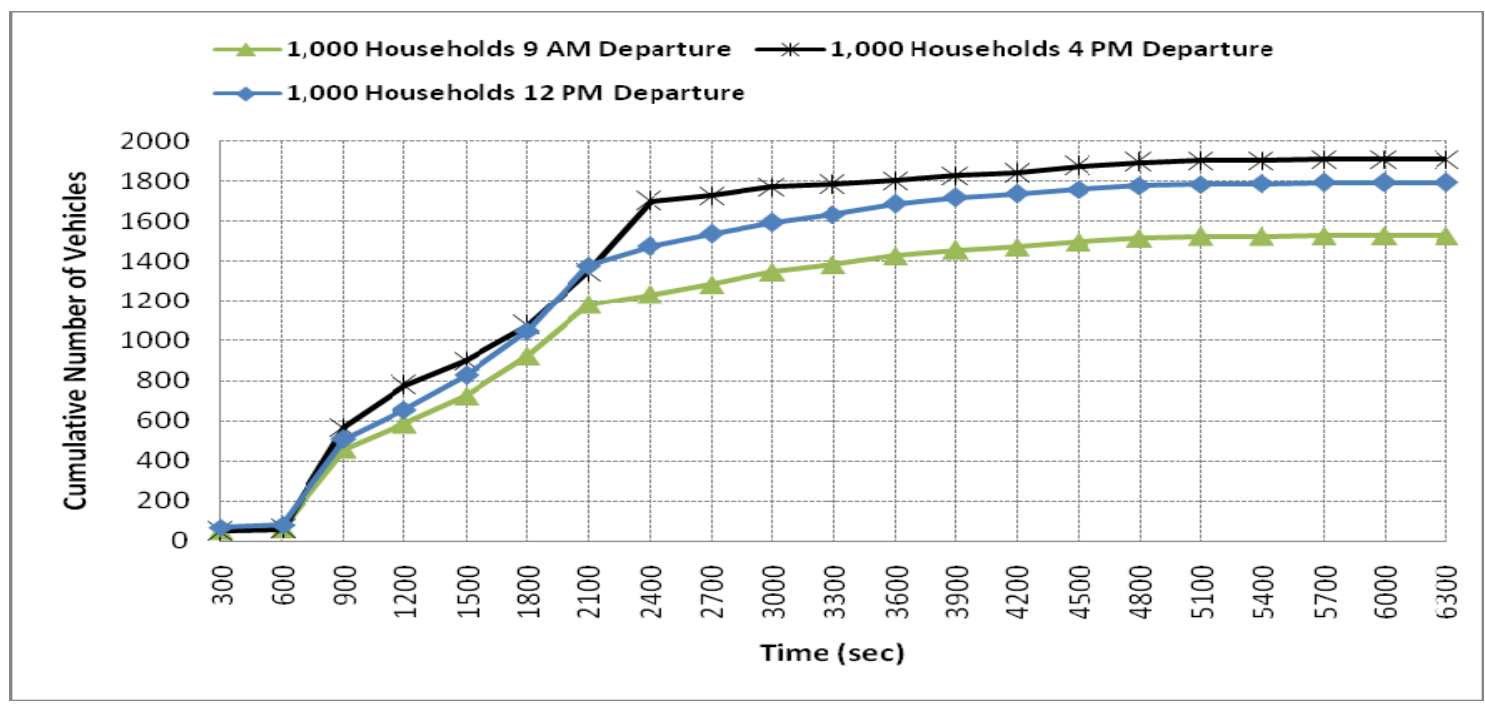

FIGURE 5: The Departure Curves for 1,000 Households at Various Times

The loading curves shown in FIGURE 4 are primarily determined by the degree of dispersion of members in the same household. The degree of location dispersion can be visualized in the figure. The departure curves depicted in FIGURE 5 are determined by the degree of dispersion and the resulting congestion level. For instance, though the numbers of vehicles loaded onto the network at time 2,100 seconds differ by 120 between 12 Noon and 4 PM, the number of vehicle departures is roughly the same. This indicates that the congestion level at $4 \mathrm{PM}$ is higher due to the heavier evacuation traffic caused by dispersed household individuals. In addition, the sampled households in this scenario generate trips massively in a few locations that cause near gridlock in the surrounding area. The high number of trips contributes to the greater congestion level as well.

Next, we conduct the time-of-day experiments on the 5,000-household scenario. Similar results can be observed in TABLE 3. The loading curves and departure curves for various times of day are depicted in FIGURE 6 and FIGURE 7 respectively.

TABLE 3: Impact of Time of Day for 5,000 Households Scenario

\begin{tabular}{|c|c|c|c|}
\hline Time & $\begin{array}{c}\text { Number of Evacuation } \\
\text { Trips }\end{array}$ & $\begin{array}{c}\text { Total System Travel } \\
\text { Time (sec) }\end{array}$ & $\begin{array}{c}\text { Network Clearance } \\
\text { Time (sec) }\end{array}$ \\
\hline \hline 9 AM & 7,414 & $10,216,626$ & 5,886 \\
\hline 12 PM & 8,851 & $12,396,487$ & 6,264 \\
\hline 4 PM & 9,726 & $14,498,627$ & 5,928 \\
\hline
\end{tabular}




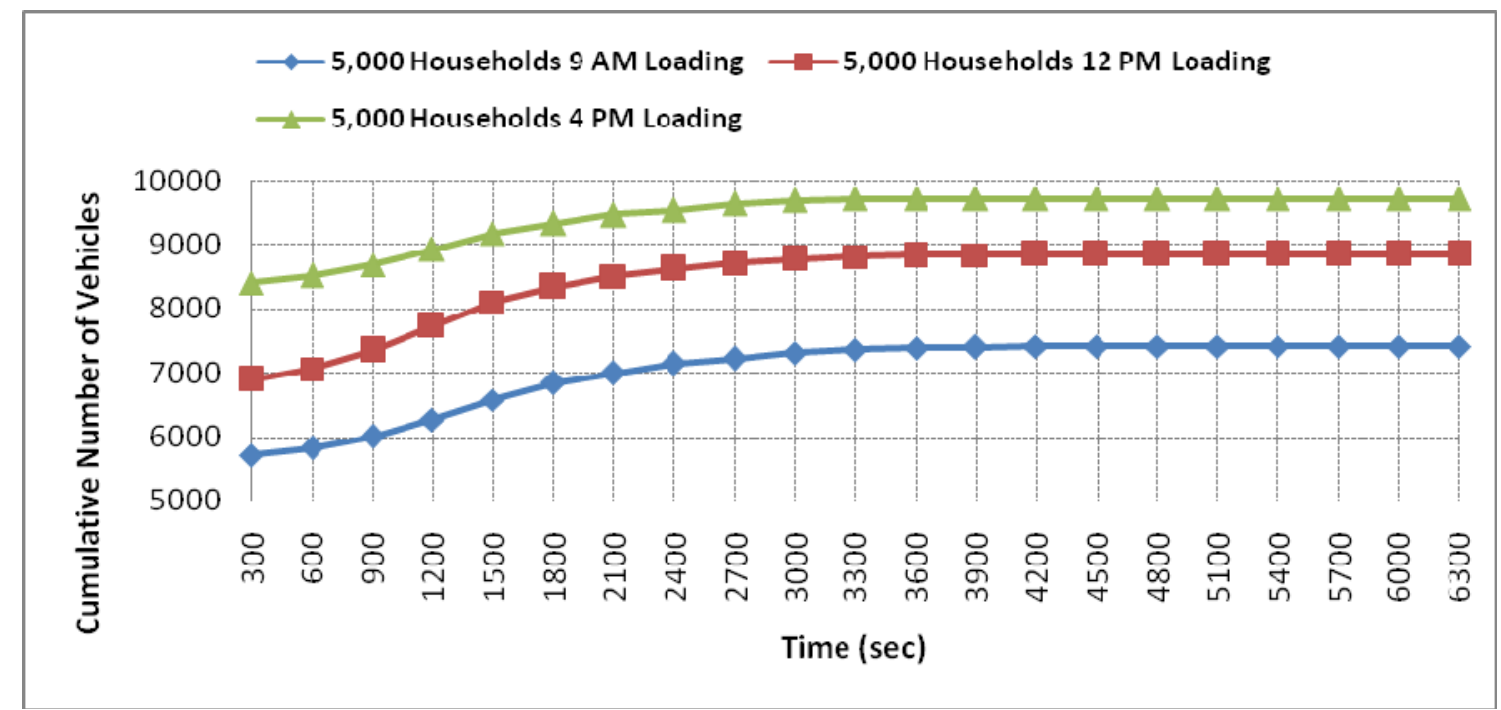

FIGURE 6: The Loading Curves for 5,000 Households at Various Times

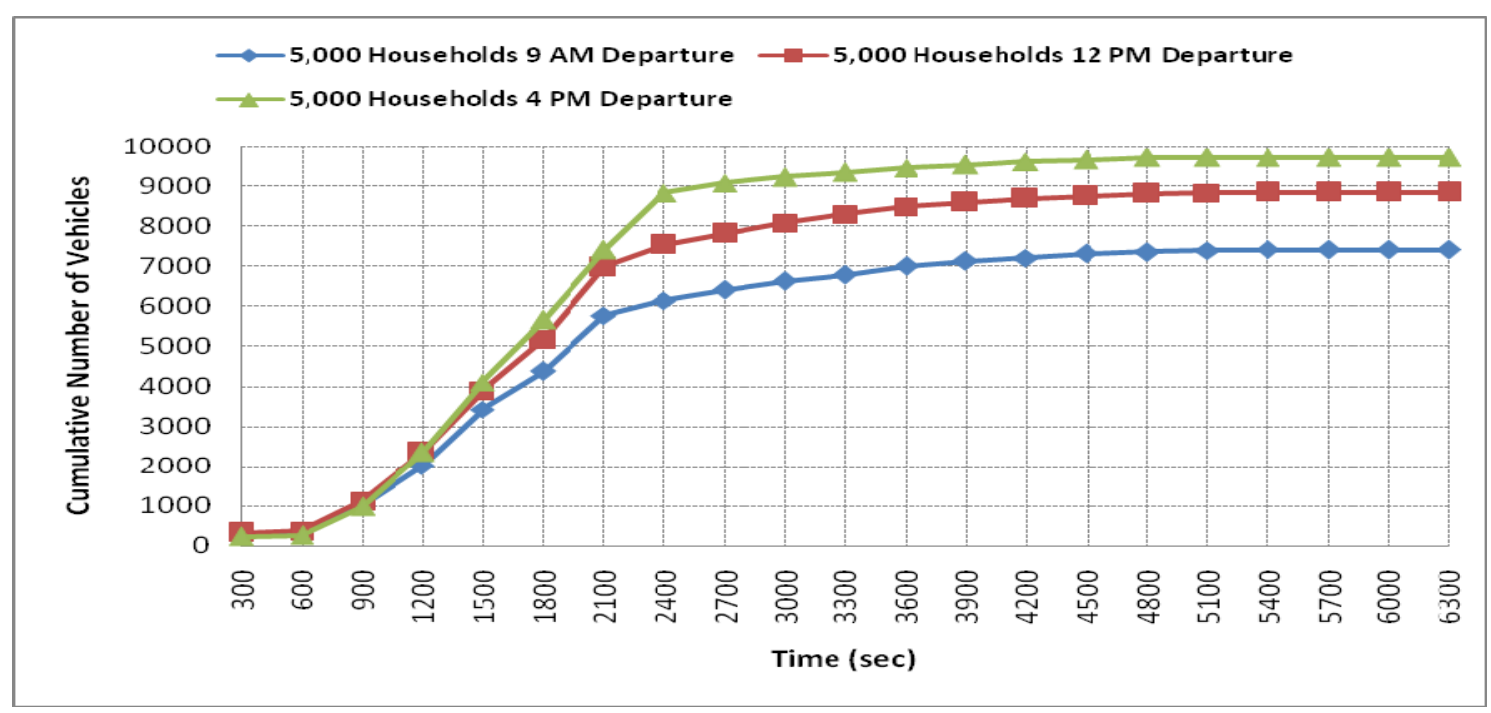

FIGURE 7: The Departure Curves for 5,000 Households at Various Times

Again, the degree of dispersion of individuals in the same household can be observed in the loading curves depicted in FIGURE 6. However, unlike the 1,000-household scenario, the number of vehicle departures remains similar only before time 1,200 seconds. The departure curve relating to the 4 PM evacuation never falls below the departure curve of 12 PM and 9AM afterward. The gridlock effect observed in the 1,000 household scenario is not apparent in this scenario.

From the experiments, we can see that evacuation system performance can be evaluated up to the second-level resolution with the proposed framework. This will be a useful tool to evaluate the time of issuing evacuation alert. For instance, one can compare the TSTT and NCT at 9:00 AM and at 9:15 AM. The decisions on traffic management or evacuation plan then can be made accordingly. 


\section{CONCLUSIONS AND FUTURE RESEARCH}

This paper develops an integrated demand-supply system to study the network repercussions of evacuation from an urban region. In the framework, an ABM model accurately positions evacuees, while a DTA model provides the time-varying traffic flow patterns. The integrated system provides a framework to analyze evacuation traffic and predict the dynamic OD trip table. In addition, the research provides a tool for evaluating network performance during evacuation.

Past research efforts have seldom considered the behavioral elements associated with the location dispersion of household members at the time of an evacuation alert, which is especially critical to the ensuing travel patterns. Without explicitly considering the additional intra-urban trips that need to be made before households evacuate out of the urban region as a single unit, a much more optimistic (and unrealistic) situation of time to evacuation would be obtained, potentially leading to misinformed policies regarding resource needs for timely evacuation.

This work addresses but one step of the evacuation analysis. A potential extension of the paper is the network design problem, including network expansion and contraflow design. One can employ the proposed framework as the evaluation function of the capacity expansion policy. 


\section{REFERENCE}

1. Perry RW, Lindell KM (2003a) Preparedness for emergency response: guidelines for the emergency planning process. Disasters 27(4):336-350.

2. Perry RW, Lindell KM (2003b) Understanding citizen response to disasters with implications for terrorism. J Contingencies Crisis Management 11(2):49-60

3. Perry, R. W., M. K. Lindell, and M. R. Greene. Evacuation Planning in Emergency Management. Lexington Books, Toronto, Ontario, Canada, 1981.

4. Johnson, N. R. Fire in a Crowded Theater: A Descriptive Investigation of the Emergence of Panic. International Journal of Mass Emergencies and Disasters, Vol. 6, No. 1, March 1988, pp. 7-26.

5. Sime, J. D. Crowd Psychology and Engineering: Designing for People or Ballbearings? In Engineering for Crowd Safety (R. A. Smith and J. F. Dickie, eds.), Elsevier Science Publishers, New York, 1993, pp. 119-131.

6. Zelinsky, W., and L. A. Kosinski. The Emergency Evacuation of Cities: A Cross-National Historical and Geographical Study. Rowman and Littlefield Publishers, Inc., 1991.

7. Murray-Tuite, P., and H. S. Mahmassani. Model of Household Trip-Chain Sequencing in an Emergency Evacuation. In Transportation Research Record: Journal of the Transportation Research Board, No. 1831, TRB, National Research Council, Washington, D.C., 2003, pp. 21-29.

8. Lin, D.-Y., Eluru, N., Waller, S.T. and Bhat, C.R. "Integration of Activity-Based Modeling and Dynamic Traffic Assignment” Transportation Research Record. 2008, (forthcoming)

9. Jones, P. M., Koppelman, F.S. and Orfeuil, J.P. “Activity Analysis: State of the Art and Future Directions” In Developments in Dynamic and Activity-Based Approaches to Travel Analysis, pp. 34-55, Gower, Aldershot, England, 1993.

10. Axhausen, K. and Gärling, T. “Activity-based Approaches to Travel Analysis: Conceptual Frameworks, Models and Research Problems” Transport Reviews, 12, pp. 324-341, 1992.

11. Kurani, K.S. and Kitamura, R. "Recent developments and the prospects for modeling household activity schedules” Report prepared for the Los Alamos National Laboratory, Institute of Transportation Studies, University of California, Davis, CA, 1996.

12. Bhat, C.R. and Koppelman F. Activity-Based Modeling for Travel Demand. Handbook of Transportation Science, R.W. Hall (ed.), Kluwer Academic Publisher, 1999.

13. Bhat, C.R., Guo, J.Y., Srinivasan, S. and Sivakumar, A. "A Comprehensive Econometric Microsimulator for Daily Activity-Travel Patterns” Transportation Research Record, Vol. 1894, pp. 57-66, 2004. 
14. Vovsha, P. and Bradley, M. "Advanced Activity-Based Models in a Context of Planning Decisions” Transportation Research Record 1981, Journal of the Transportation Research Board, TRB, National Research Council, Washington, D.C., pp. 34-41, 2005.

15. Pinjari, A., Eluru, N., Copperman, R., Sener, I.N., Guo, J.Y., Srinivasan, S. and Bhat, C.R. "Activity-Based Travel-Demand Analysis for Metropolitan Areas in Texas: CEMDAP Models, Framework, Software Architecture and Application Results” Report 4080-8, prepared for the Texas Department of Transportation, 2006.

16. Bowman, J. L., Bradley, M. A., Shiftan, Y., Lawton, T. K. and Ben-Akiva, M. E. "Demonstration of an Activity Based Model System for Portland", 8th World Conference on Transport Research, July 12-17, Antwerp, Belgium, 1998.

17. Vovsha P., Petersen, E. and Donnelly, R. "Micro-Simulation in Travel Demand Modeling: Lessons Learned from the New York Best Practice Model” Transportation Research Record 1805, pp. 68-77, 2002.

18. Vovsha, P., Petersen, E. and Donnelly R. "Impact of Intra-Household Interactions on Individual Daily Activity-Travel Patterns" Presented at the 83rd Annual Meeting of the Transportation Research Board, Washington, D.C., 2004.

19. Bowman, J. L., and Bradley, M. A. “Activity-Based Travel Forecasting Model for SACOG: Technical Memos Numbers 1-11” 2005-2006, Available at http://jbowman.net.

20. Bradley, M., Outwater, M., Jonnalagadda, N., and Ruiter, E. "Estimation of an ActivityBased Micro-Simulation Model for San Francisco". Presented at the 80th Annual Meeting of the Transportation Research Board, Washington D.C., 2001.

21. Waller, S.T. and Ziliaskopoulos, A.K. "A Visual Interactive System for Transportation Algorithms" Presented at the 78th Annual Meeting of the Transportation Research Board, Washington, D.C, 1998.

22. Taylor, N. B. “CONTRAM 5: an Enhanced Traffic Assignment Model” TRLL Research Report 249, 1990.

23. Ben-Akiva, M., Bierlaire, M., Koutsopoulos, H. and Mishalani, R. "DynaMIT: a Simulationbased System for Traffic Prediction and Guidance Generation” Paper presented at TRISTAN III, 1998.

24. Ben-Akiva, M., Bierlaire, M., Koutsopoulos, H. and Mishalani, R. "DynaMIT: a Simulationbased System for Traffic Prediction” Paper presented at the DACCORD Short Term Forecasting Workshop, 1998.

25. Massachusetts Institute of Technology "DTA System Enhancement and Evaluation at Traffic Management Center, Task P: Framework for the Use of DynaMIT 145 in Transportation Planning Applications (DynaMIT-P)” Technical report, Submitted to Oak Ridge National Laboratories, 2000. 
26. Mahmassani, H. S., Hu, T-Y. and Jayakrishnan, R. "Dynamic Traffic Assignment and Simulation for Advanced Network Informatics (DYNASMART)” In Proceedings of the 2nd International CAPRI Seminar on Urban Traffic Networks, Capri, Italy, 1992.

27. Guo, J.Y., and Bhat, C.R. "Population Synthesis for Microsimulating Travel Behavior" Forthcoming, Transportation Research Record, 2006.

28. Eluru, N., A.R. Pinjari, J.Y. Guo, I.N. Sener, S. Srinivasan, R.B. Copperman, and C.R. Bhat, "Population Updating System Structures and Models Embedded Within the Comprehensive Econometric Microsimulator for Urban Systems (CEMUS)," forthcoming, Transportation Research Record 2008

29. Ziliaskopoulos, A. K., and Lee, S. “A Cell Transmission Based Assignment-simulation Model for Integrated Freeway/surface Street Systems” Presented at the 75th Annual Meeting of the Transportation Research Board, Washington, D.C, 1996.

30. Daganzo, C. F. “The Cell Transmission Model: A Simple Dynamic Representation of Highway Traffic Consistent with the Hydrodynamic Theory” Transportation Research Part B: Methodology 28B, pp. 269-287, 1994.

31. Daganzo, C. F. “The Cell Transmission Model, Part II: Network Traffic” Transportation Research Part B: Methodology 29B, pp.79-93, 1995.

32. Ziliaskopoulos, A.K. and Mahmassani, H.S. "A Time Dependent Shortest Path Algorithm for Real-time Intelligent Vehicle/highway Systems" Transportation Research Record 1408, pp.94-104, 1993.

33. Ziliaskopoulos, A.K. and Mahmassani, H.S. “A Note on Least Time Path Computations Considering Delays and Prohibitions for Intersection Movements” Transportation Research Part B, 30(5), pp. 359-367, 1996.

34. Ziliaskopoulos, A.K. and Kotzinos, D. “A Massively Parallel Time-dependent Least-timepath Algorithm for Intelligent Transportation Systems Applications” Comput. Aided Civ. Infrastruct. Eng. 16 (5) (2001) 337 -346 (Special Issue: High Performance Computing

35. Chang, E. J. “Time-Varying Intermodal Person Trip Assignment” Ph.D. Dissertation, Northwestern University, 2004.

36. Ziliaskopoulos, A.K., Waller, S.T., Li, Y. and Byram, M. "Large Scale Dynamic Traffic Assignment: Implementation Issues and Computational Analysis," Journal of Transportation Engineering Volume 130, No. 5, pp. 585-593, 2004.

37. Ziliaskopoulos, A.K. and Waller, S.T. "An Internet Based Geographic Information System that Integrates Data, Models, and Users for Transportation Applications" Transportation Research Part C Vol. 8 pp. 427-444, 2000. 
Lin, Eluru, Waller and Bhat

38. Pendyala, R.M., R. Kitamura, A. Kikuchi, T. Yamamoto, and S. Fujii (2005) FAMOS: The Florida Activity Mobility Simulator. Transportation Research Record 1921, Journal of the Transportation Research Board, TRB, National Research Council, Washington, D.C., pp. 123-130. 